\title{
The Basement Membrane Controversy
}

Dear Sir:

I have read with interest the Editorial Articles by yourself, Drs. Gunderson et al., and Siperstein et al. in Diabetologia (Nov. 1978). I think this is an excellent approach to important controversial issues.

Although I do not intend to take sides in this controversy, I would like to offer a few caveats in relation to some of the statements made by Siperstein's group in this and previous publications. These deal with the need to take into consideration the heterogeneity of diabetes when performing studies of microangiopathy, and with the use of the concept of prediabetes.

First, there is now a large body of evidence of clinical, epidemiological, biochemical, and immunogenetic nature, clinching the concept that insulin independent (IID) and insulin dependent (IDD) diabetes are different diseases. Although we do see retinopathy and other manifestations of microangiopathy in both, we must seriously consider the possibility of different pathogenesis for these complications in the two different types of diabetes. Indeed, we have evidence from our laboratory that even within strictly defined IDD, severe retinopathy is associated with some of the HLA antigens only for particular patient subsets according to age at onset of disease [1]. This seems to suggest that the pathogenesis of microangiopathy within IDD may be genetically heterogeneous. Inspection of Table IV in Siperstein's original publication [2], reveals a great heterogeneity in both the age of the patients and their mode of treatment. At least $29(57 \%)$ were not on insulin and probably many more were IID patients. Furthermore, many of the younger ones, often not on insulin, may have had maturity onset hyperglycaemia in the young [5]. Many patients were black, which further complicates the problem of heterogeneity; these patients may have a type of hyperglycaemia akin to maturity onset hyperglycaemia of the young [6] described in Caucasians. Any studies of microangiopathy should be conducted only on carefully selected and defined patients, belonging to one discrete type of diabetes. Conjugal diabetes, studied by Siperstein et al. [2], is almost invariably of the IID type and, therefore, the data found from the studies of their offspring may be irrelevant to insulin dependent diabetics.

Secondly, IID is itself most likely genetically heterogeneous, and there is no evidence for an autosomal recessive mode in this disease. Siperstein's group has recently suggested that all diabetes is autosomal recessive [3]. As previously implied [4], that is akin to saying that the earth is flat. Confirming the view of extensive heterogeneity in IID, Tattersall et al. [7] when studying 37 conjugal diabetic families, found the prevalence of diabetes among the offspring to vary from zero to nearly $100 \%$, strongly suggesting that more than one genotype was involved. I feel, therefore, that it is misleading to refer to the offspring of conjugal diabetics as prediabetics. For the moment, unfortunately, we do not have a marker for prediabetes. In fact, this applies to both IDD and IID. It would be best, at least for Caucasians, to defer the use of this term until we learn how to identify diabetic genotypes before the phenotypes are apparent, regardless of the patient's family history. All research in diabetes from now on should take into consideration the probably extensive heterogeneity which characterizes this symptom-complex.

\section{References}

1. Barbosa, J., et al.: Diabetic proliferative retinopathy and the HLA. (Submitted for publication)

2. Siperstein, M. D., Unger, R. H., Madison, L. L.: Studies of mus- 
cle capillary basement membrane in normal subjects, diabetic and prediabetic patients. J. Clin. Invest. 47, 1973-1999 (1968)

3. Rosenthal, M. B., Goldfine, I. D., Siperstein, M. D.: Inheritance of diabetes mellitus. N. Engl. J. Med. 295, 1321 (1976)

4. Rimoin, D., Rotter, J.: Inheritance of diabetes mellitus. N. Engl. J. Med. 295, 1321 (1976)

5. Barbosa, J., Ramsay, R., Goetz, F. C.: Plasma glucose, insulin, glucagon, and growth hormone in kindreds with maturity-onset type of hyperglycemia in young people. Ann. Intern. Med. 88, 595-601 (1978)

6. MacDonald, M.: Lower frequency of diabetes among hospitalized Negro than white children. Acta Genet. Med. Gemellol. 24, 119-126 (1975)
7. Tattersall, R., Fajans, S.: Prevalence of diabetes in 199 offspring of 37 conjugal diabetic parents. Diabetes 24, 452-462 (1975)

Jose Barbosa, M. D.

Division of Endocrinology and Metabolism

Department of Medicine

Mayo Memorial Building

420 Delaware St. SE.

Minneapolis, MN 55455

USA
Dear Sir:

In their editorial [1] Dr. Lundbaek and his colleagues have published, for the first time, a subset of the data from the NIH study concerning muscle capillary basement membrane thickening in Pima Indian diabetics, the nondiabetic offspring of two non-diabetic parents, and the non-diabetic offspring of two diabetic parents, which were presented at the 1976 Clinical Research Meetings [2].

Following a brief discussion of these data, they quote out of context, a statement made by Siperstein [3] that "In summary data from this (Siperstein's) laboratory and from the National Institutes of Health cooperative study would support the conclusion that diabetic microagniopathy does not result from the hyperglycemia or the hypoinsulinemia of diabetes mellitus." This statement represents Dr. Siperstein's interpretation of his own and these preliminary data. Lundbaek and his colleagues then go on to state that "the endorsing of such a statement and all of its implications by the NIH is - somewhat surprising." In addition to being surprising, this statement is erroneous, since no endorsement of this conclusion has ever been made by the National Institutes of Health. In the subsequent editorial by Siperstein et al. [4], some additional results are cited, which suggest contrary conclusions to those suggested by Lundbaek, illustrating a possible alternative interpretation, as well as illustrating the complexity of analyzing such data. In both editorials the numerical data are correct, and are based on the same, but incomplete results. Although data acquisition is now com- plete, analysis of the NIH study is still in progress. Thus any final interpretations or conclusions preceding publication of the complete analysis are premature. Thus, in the meantime the question of whether or not the Pima Indian muscle capillary membrane data are sufficient and adequate to refute or support the hypothesis espoused by Lundbaek and others, or the alternate, as restated by Siperstein, cannot be judged, since only preliminary (and incomplete) evidence has yet been presented.

\section{References}

1. Gundersen, H. J. G., Osterby, R., Lundbaek, K.: The basement membrane controversy. Diabetologia 15, 361-363 (1978)

2. Aronoff, S. L., Bennett, P.H., Williamson, J.R., Siperstein, M.D., Plumer, M. E., Miller, M.: Muscle capillary basement membrane (MCBM) in prediabetic, diabetic, and normal Pima Indians and Causasians (Abstract). Clin. Res. 24, 455 A (1976)

3. Siperstein, M. D.: The current status of quadriceps capillary basement membrane thickening in diabetes and pre-diabetes. In: Colloque International Biologie et Pathologie des Membranes Basales, Paris, October 1977, Abstract

4. Siperstein, M. D., Feingold, K. R., Bennett, P. H.: Hyperglycemia and diabetic microangiopathy. Diabetologia 15, 365-367 (1978)

Peter H. Bennett, M. B., M.R.C.P., F.F.C.M.

Epidemiology and Field Studies Branch

National Institute of Arthritis,

Metabolism and Digestive Diseases

1440 East Indian School Road

Phoenix, AZ 85014

USA 